\title{
Evaluation passenger car unit for motorcycle in Indonesia Highway Capacity Manual (Case study: Bandung and Semarang)
}

\author{
Najid $^{1, *}$ \\ ${ }^{1}$ Tarumanegara University, Department Civil Engineering, Jakarta, Indonesia
}

\begin{abstract}
Value of Passenger Car Unit or commonly known as PCU value is a value that is given to any vehicle that is classified into heavy vehicles, light vehicles (passenger car) and motorcycles. The value of passenger car unit on Indonesia Highway Capacity Manual (IHCM) set up in 1997 is based on a study conducted from 1980-1990 in several cities in Indonesia At the time of the study, the traffic conditions are very different to the current traffic conditions. That affects of difference traffic conditions are the composition of traffic, traffic regulations, traffic density, traffic discipline and the presence of mass transit, so that the results of traffic analysis do not always correspond to reality as there are anomalies in the determination of the level of road service (Najid, 2014). As well the incompatibility of the capacity value which is considered due to the incompatibility value of Passenger Car Units (PCU). Evaluation PCU become very important to get the value of traffic parameters into compliance with actually occur. In accordance with the traffic density is higher actually, then it is necessary to study for evaluation against PCU current value and the need to approach or to get the value of PCU more in line with current traffic conditions. Data collected at two cities, those are Bandung and Semarang. Based on analysis found PCU's value that got from survey have difference but not all significantly with PCU value in IHCM.
\end{abstract}

\section{Background}

As we know that actually the use of private transport is very high, especially motorcycles in almost cities in Indonesia, especially the cities in Java Island. Those phenomenon caused by rapidly growth of private vehicle usage especially motor cycle, while on some routes passengers of public transport decreased significantly. This shows the displacement of demand from public transport to private transport.

The high usage of private transport that cause in-efficiency of urban transportation, so the level of road service to be down [1]. The fall in the level of road service is an indicator that can be used by the Government in road development planning. The problem is in the analysis of the results obtained often not appropriate like traffic volume is greater than

* Corresponding author: najid2009@yahoo.co.id 
capacity so that ratio of volume and capacity be more than one. Therefore, the determination of the value of passenger car unit which is the decisive factor in the calculation of traffic volume becomes very important to be evaluated.

The calculation of road capacity in IHCM using passenger car unit that strongly influenced by the condition or characteristics of the vehicle, traffic as well as by the characteristics of the road it self based on its function. So in this research need survey conducted in various road type.

This research undertaken Bandung city and Semarang city as case study, so the survey have conducted on those cities.

\section{Research objectives}

This research aims to :

- Determining the value of passenger car unit based on survey result.

- Identify relationships between types of road with the passenger car unit values.

- Formulate the magnitude of the mismatch between the value of passenger car unit based on survey and based IHCM.

\section{Problem Identification and Limitation}

- Value of PCU in IHCM look too optimistic and rude.

- Value of PCU in IHCM not consider the actual service conditions.

- PCU value that evaluated and analyzed is PCU value for urban roads.

\section{Review of literature}

Directorate General Bina Marga has recorded the total length of the existing road network in Indonesia, which reached $376176 \mathrm{~km}$ consisting toll road $741.97 \mathrm{~km}(0.20 \%)$, non-toll national roads $38569 \mathrm{~km}(10.25 \%)$, road provinces $48681 \mathrm{~km}(12.94 \%)$, district road 255 $253 \mathrm{~km}(67.85 \%)$ and urban road $32932 \mathrm{~km}(8.75 \%)$. In-efficiency traffic movement take place on urban roads in everyday [2].

As stated in the background the growth of private vehicle is cause declining the level of service in the mark with declined travel speed and increased the density of traffic on the road. The calculation of traffic volume is highly influenced by the value of PCU (Passenger Car Unit). Value of PCU is the value of equality of another vehicle to vehicle passenger cars were categorized as light vehicle (LV), while other vehicles are motorcycles (MC) and heavy vehicles (HV) including all medium bus and big bus as well as all the trucks.

The Directorate General of Highways, Ministry of Public Works in cooperation with the consultant at that time Sweroad of Sweden make traffic's handbook named Indonesian Highway Capacity Manual (IHCM) in year 1997. The manual has been made for determination of the PCU value of vehicles based on traffic conditions and the road conditions. PCU value of the vehicle is also distinguished in urban areas or in areas between cities. The following Table 1a-1c shows the value for the urban PCU based IHCM [3]:

Table 1a. Intersection

\begin{tabular}{|l|c|c|}
\hline \multicolumn{1}{|c|}{ Vehicle Type } & PCU Protected & PCU Opposed \\
\hline Light Vehicle (LV) & 1 & 1 \\
\hline Heavy Vehicle (HV) & 1.3 & 1.3 \\
\hline Motor Cycle (MC) & 0.2 & 0.4 \\
\hline
\end{tabular}


Table 1b. Urban road (undivided road)

\begin{tabular}{|c|c|c|c|c|}
\hline \multirow{4}{*}{ Road Type } & \multirow{4}{*}{$\begin{array}{l}\text { Traffic Flow } \\
2 \text { ways } \\
\text { (vehicle/hour) }\end{array}$} & \multicolumn{3}{|c|}{ PC } \\
\hline & & \multirow{3}{*}{ HV } & $\mathrm{MC}$ & \\
\hline & & & \multicolumn{2}{|c|}{ Road Width, Wc (m) } \\
\hline & & & $\leq 6$ & 6 \\
\hline \multirow{2}{*}{$\begin{array}{l}2 \text { lanes undivided } \\
(2 / 2 \mathrm{UD})\end{array}$} & $0-1799$ & 1.3 & 0.50 & 0.40 \\
\hline & $>1800$ & 12 & 0.35 & 0.25 \\
\hline \multirow{2}{*}{$\begin{array}{l}4 \text { lanes undivided } \\
\text { (4/2 UD) }\end{array}$} & $0-699$ & 1.3 & \multicolumn{2}{|c|}{0.40} \\
\hline & $\geq 3700$ & 1.2 & \multicolumn{2}{|c|}{0.25} \\
\hline
\end{tabular}

Table 1c. Urban road (divided road)

\begin{tabular}{|l|c|c|c|}
\hline \multicolumn{1}{|c|}{ Road Type } & Traffic Flow/lane & \multicolumn{2}{c|}{ PC } \\
\cline { 3 - 4 } & (Vehicl/hour) & HV & MC \\
\hline 2 lanes 1 Way (2/1) & $0-1049$ & 1.3 & 0.40 \\
4 lanes 2 Ways (4/2 D) & $\geq 1050$ & 1.2 & 0.25 \\
\hline 3 lanes 1 Way (3/1) & $0-1099$ & 1.3 & 0.40 \\
6 lanes 2 Ways (6/2 D) & $\geq 1100$ & 1.2 & 0.25 \\
\hline
\end{tabular}

The magnitude of the dimensions of the vehicle will affect the value of PCU [4]. Research to determine the value of PCU been done by some researchers. either on the road section and at signalized intersection or intersection is not signalized. Past research in Surakarta by Rosma in determining PCU at signalized intersections. Value of motorcycle's PCU was determined with linear regression method, result of the research a value of motorcycle's PCU between 0.11 to 0.2. As for bus passengers's PCU between 1.02 to 1.72 [5].

As comparisson total population and density population in Bandung and Semarang showed on table 1d follow:

Table 1d. Population comparisson

\begin{tabular}{|c|c|c|c|c|}
\hline No & City & Population (habitant) & Area $\left(\mathrm{km}^{2}\right)$ & Density habitant $/ \mathrm{km}^{2}$ \\
\hline 1 & Bandung & 2.341 .097 & 167,67 & $13.962,5$ \\
\hline 2 & Semarang & 1.622 .520 & 373,78 & $4.340,8$ \\
\hline
\end{tabular}

Source: Central Statistic Bureau (2015) [6]

\section{Methodology}

Figure 1 : Methodology

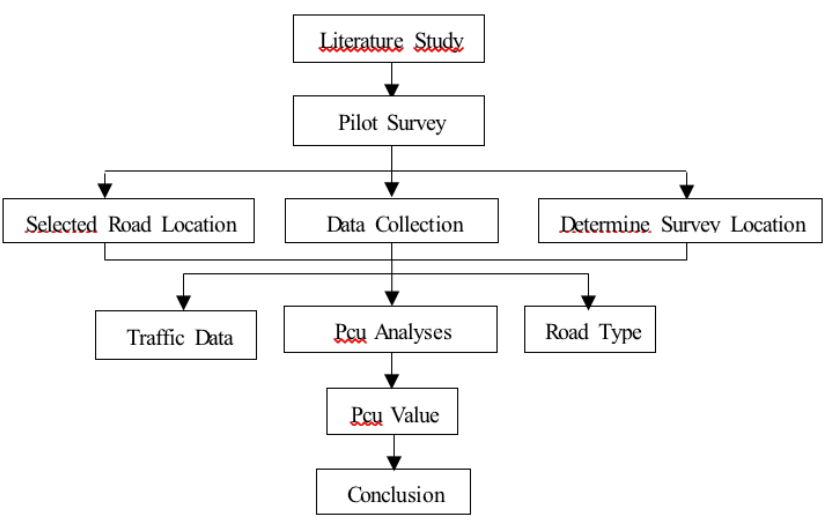


Flow chart showing the systematic study of the prose of this research that can be seen in Figure 1.

\section{Determining factor passenger car unit}

To determine PCU value, be used headway's method [7]. In this method, PCU various types of vehicles on the certain condition at certain road type calculated by headway's method. Estimated vehicles $1,2,3, \ldots \ldots \ldots \ldots$, $\mathrm{i}$, with the surveyed time of $\mathrm{t} 1, \mathrm{t} 2, \mathrm{t} 3$..........., ti, is calculated as the vehicle passed the imaginer line. First Vehicle movement that passed the imaginer line be noted its time and the following vehicle be noted its time too. The difference of time is a headway. Do the same procedure for any following vehicle. The following vehicle consists of passenger car - motor cycle, motor cycle-passenger car, passenger car-passenger car and motor cycle-motor cycle.

$\mathrm{PCU}=((\mathrm{HSM}-\mathrm{Q}) / \mathrm{NSM}) /(\mathrm{HCC}-\mathrm{Q} / \mathrm{NCC})$

Or

PCU.Sm $=$ HAA $/$ HCC

with:

$\overline{\mathrm{HSM}}=$ Headway between motorcycle

HAA $=$ Headway between motorcycle and car

$\mathrm{HCC}=$ Headway between car

$\mathrm{Q}=$ Correction Factor

$\mathrm{NSM}=$ total HSM

$\mathrm{NCC}=$ total $\mathrm{HCC}$

\section{Data collection}

The flow of traffic on all roads are relatively high, especially at peak hour. The following road geometric data for the survey areas in Bandung:

- Pasteur road has a total road width of 23 metre with a median width of 0.5 metre and width of sidewalks on both sides of road each 2.0 metres, represents 6/2 D (Divided) and intensive business location

- Dipati Ukur road whole road width is 10 metres, with a median width of 0.5 metre and width of sidewalks on both sides of the road each 1.5 metres, represent 4/2 UD (Undivided) and solid business location

- Juanda road in whole width of the road is 26 metres, with a median width of 0.5 metre and width of sidewalks on both sides of road each 2.0 metres, represent $4 / 2$ D and solid business location

- Tubagus Ismail road whole width of the road is 9 metres, without median and width of sidewalks on both sides of the road each 1.5 metres, represent 2/2 UD and residential location

The following road geometric data for the survey areas in Semarang :

- Thamrin road has a total width of 18,5 metres with a median width of 0.5 metre and width of sidewalks on both sides of road each 2.0 metres, represent $4 / 2 \mathrm{D}$ and office location.

- Soegijopranata road has a total width of 25 metres without median and width of sidewalks on both sides of road each 2.0 metres, represent 6/2 UD and office location. 
- Imam Bonjol road has a total width of 18 metres without median and width of sidewalks on both sides of road each 2.0 metres and 4/2 UD and business location

- Pandanaran road has total width 33 metres with a median width of 1.0 metre and width of sidewalks on both sides of road each 2.0 metres, represent $6 / 2 \mathrm{D}$ and office location.

As comparison population in Bandung is 2,470,802 person and population in Semarang. From the data survey shows that the number of motorcycles is quite high compared to other type of vehicles.

\subsection{Data collection for PCU of motorcycles}

Table 2.1. Dipati Ukur Road 2/2 UD

\begin{tabular}{|c|c|c|c|c|c|c|}
\hline Periode & $\mathrm{Ta}$ & Tc & $\mathrm{Td}$ & $\mathrm{Tb}$ & ta & tb \\
\hline $7.30-8.00$ & $\mathrm{Mb}-\mathrm{Mb}$ & Mb-Mt & $\mathrm{Mt}-\mathrm{Mb}$ & Mt-Mt & $X i-\mu$ & $\mathrm{Xi}-\mu$ \\
\hline 1 & 2.63 & 1.1 & 1.41 & 1.14 & 0.504 & 0.029 \\
\hline 2 & 2.21 & 1.23 & 1.49 & 1 & 0.084 & 0.111 \\
\hline 3 & 2.07 & 1.02 & 1.62 & 1.2 & 0.056 & 0.089 \\
\hline 4 & 1.58 & 2.17 & 1.08 & 1.16 & 0.546 & 0.049 \\
\hline 5 & 2.33 & 1.62 & 1.29 & 1.1 & 0.204 & 0.011 \\
\hline 6 & 1.61 & 1.07 & 2.81 & 1.05 & 0.516 & 0.061 \\
\hline 7 & 2.27 & 1.11 & 2.14 & 1.1 & 0.144 & 0.011 \\
\hline 8 & 2.03 & 0.97 & 1.24 & 1.07 & 0.096 & 0.041 \\
\hline 9 & 2.31 & 1.51 & 1.42 & 1.12 & 0.184 & 9E-03 \\
\hline 10 & 2.22 & 1.07 & 3.8 & 1.17 & 0.094 & 0.059 \\
\hline$\sum$ & 21.26 & 12.87 & 18.3 & 11.11 & 1.394 & 0.289 \\
\hline \multirow[t]{2}{*}{$\mu$} & 2.126 & 1.287 & 1.83 & 1.111 & & \\
\hline & & & & & ta & tb \\
\hline $9.00-9.30$ & $\mathrm{Ta}$ & Tc & $\mathrm{Td}$ & $\mathrm{Tb}$ & $\mathrm{Xi}-\mu$ & $\mathrm{Xi}-\mu$ \\
\hline 1 & 2.47 & 1.38 & 3.02 & 1.04 & 0.90 & 0.13525 \\
\hline 2 & 2.40 & 2.15 & 2.24 & 1.82 & 0.84 & 0.64475 \\
\hline 3 & 2.60 & 2.61 & 1.26 & 1.35 & 1.04 & 0.17975 \\
\hline 4 & 1.00 & 1.01 & 2.21 & 1.07 & 0.56 & 0.10025 \\
\hline 5 & 1.12 & 1.10 & 1.16 & 1.14 & 0.44 & 0.03275 \\
\hline 6 & 1.09 & 0.97 & 1.08 & 1.10 & 0.469 & 0.07525 \\
\hline 7 & 1.09 & 3.06 & 1.18 & 1.16 & 0.469 & 0.01525 \\
\hline 8 & 1.45 & 1.68 & 2.45 & 0.99 & 0.109 & 0.18525 \\
\hline 9 & 0.99 & 2.85 & 2.90 & 1.05 & 0.569 & 0.12025 \\
\hline 10 & 1.40 & 1.04 & 1.67 & 1.01 & 0.162 & 0.16025 \\
\hline$\Sigma$ & 15.62 & 17.83 & 19.13 & 11.70 & 3.785 & 1.09275 \\
\hline$\mu$ & 1.562 & 1.783 & 1.91 & 1.17 & & \\
\hline
\end{tabular}


Data collection techniques on the road is use simple tools like the regular stopwatch, pen and note's board. Because many factors can affect the accuracy of the data and usually the human factor is the most decisive. The survey result show on the tables 2.1-2.4 (Bandung) and 2.5-2.8 (Semarang) as show on table.

Table 5.1 (road 2/2 UD) the headway car and motorcycle (Tc), motorcycle and car (Td) and motorcycle and motorcycle (Tb) in the morning (peak hour) is rather similar than during the day except Ta and Tc, the analysis shows the traffic volume in the morning is not significantly different with during the day.

Table 2.2. Juanda Road 4/2 D

\begin{tabular}{|c|c|c|c|c|c|c|}
\hline Periode & $\mathrm{Ta}$ & $\mathrm{Tc}$ & $\mathrm{Td}$ & $\mathrm{Tb}$ & ta & $\mathrm{tb}$ \\
\hline $7.30-8.00$ & $\mathrm{Mb}-\mathrm{Mb}$ & $\mathrm{Mb}-\mathrm{Mt}$ & Mt-Mb & Mt-Mt & $\mathrm{Xi}-\mu$ & $\mathrm{Xi}-\mu$ \\
\hline 1 & 6.98 & 1.20 & 2.12 & 1.14 & 2.823 & 0.285 \\
\hline 2 & 3.62 & 1.10 & 0.74 & 0.75 & 0.537 & 0.675 \\
\hline 3 & 4.46 & 1.53 & 2.43 & 1.20 & 0.303 & 0.225 \\
\hline 4 & 3.87 & 3.26 & 1.62 & 1.41 & 0.282 & 0.015 \\
\hline 5 & 4.10 & 2.43 & 1.94 & 1.35 & 0.057 & 0.075 \\
\hline 6 & 2.48 & 1.31 & 4.22 & 1.05 & 1.677 & 0.375 \\
\hline 7 & 4.16 & 1.22 & 3.21 & 3.53 & 0.003 & 2.1 \\
\hline 8 & 5.00 & 1.46 & 0.36 & 1.58 & 0.843 & 0.15 \\
\hline 9 & 2.06 & 2.27 & 2.13 & 0.83 & 2.097 & 0.6 \\
\hline 10 & 4.83 & 1.61 & 5.70 & 1.43 & 0.678 & 0.16 \\
\hline$\sum$ & 41.52 & 17.355 & 24.45 & 14.25 & 4.002 & 1.275 \\
\hline$\mu$ & 4.152 & 1.7355 & 2.445 & 1.425 & & \\
\hline $9.00-9.30$ & $\mathrm{Ta}$ & Tc & $\mathrm{Td}$ & $\mathrm{Tb}$ & $\mathrm{Xi}-\mu$ & $\mathrm{Xi}-\mu$ \\
\hline 1 & 3.24 & 1.84 & 4.02 & 1.58 & 0.13 & 0.278 \\
\hline 2 & 1.2 & 2.86 & 2.98 & 2.42 & 2.17 & 0.562 \\
\hline 3 & 3.3 & 3.48 & 1.14 & 1.80 & 0.07 & 0.058 \\
\hline 4 & 3.86 & 1.34 & 2.94 & 0.96 & 0.49 & 0.898 \\
\hline 5 & 2.56 & 1.46 & 1.84 & 2.98 & 0.81 & 1.122 \\
\hline 6 & 3.82 & 1.26 & 0.9 & 1.46 & 0.45 & 0.398 \\
\hline 7 & 3.8 & 4.08 & 1 & 1.54 & 0.43 & 0.318 \\
\hline 8 & 4.06 & 2.24 & 3.26 & 2.62 & 0.69 & 0.762 \\
\hline 9 & 3.8 & 3.8 & 3.86 & 1.54 & 0.43 & 0.318 \\
\hline 10 & 4.06 & 1.12 & 2.22 & 1.68 & 0.69 & 0.178 \\
\hline$\sum$ & 33.7 & 23.48 & 24.16 & 18.58 & 3.67 & 2.918 \\
\hline$\mu$ & 3.37 & 2.348 & 2.416 & 1.858 & & \\
\hline
\end{tabular}

Table 2.2 (road 4/2 D) the headway between the car (Ta), car and motorcycle (Tc), motorcycle and car (Td) and motorcycle and motorcycle (Tb) in the morning (peak hour) is smaller than during the day but data deviation is higher, the analysis shows the traffic volume in the morning is rather similar with during the day. 
Table 2.3. Tubagus Ismail Road 2/2 UD

\begin{tabular}{|c|c|c|c|c|c|c|}
\hline Periode & $\mathrm{Ta}$ & tc & $\mathrm{Td}$ & $\mathrm{Tb}$ & ta & $\mathrm{Tb}$ \\
\hline $7.30-8.00$ & $\mathrm{Mb}-\mathrm{Mb}$ & $\mathrm{Mb}-\mathrm{Mt}$ & Mt-Mb & Mt-Mt & $X i-\mu$ & $\mathrm{Xi}-\mu$ \\
\hline 1 & 2.7 & 4.8 & 4.6 & 1.6 & 1.78 & 0.18 \\
\hline 2 & 4.4 & 3.8 & 2.4 & 1.3 & 0.08 & 0.12 \\
\hline 3 & 7.8 & 6.2 & 7.2 & 1.4 & 3.32 & 0.02 \\
\hline 4 & 4.8 & 3.2 & 4.8 & 1.2 & 0.32 & 0.22 \\
\hline 5 & 3.8 & 5.2 & 7.4 & 1.2 & 0.68 & 0.22 \\
\hline 6 & 4.9 & 5.2 & 6.6 & 2.1 & 0.42 & 0.68 \\
\hline 7 & 6.2 & 4.6 & 8.4 & 1.4 & 1.72 & 0.02 \\
\hline 8 & 4.8 & 8.2 & 4.4 & 1.2 & 0.32 & 0.22 \\
\hline 9 & 2.2 & 3.6 & 5.2 & 1.3 & 2.28 & 0.12 \\
\hline 10 & 3.2 & 4.2 & 2.8 & 1.5 & 1.28 & 0.08 \\
\hline$\sum$ & 44.80 & 49.00 & 53.80 & 14.20 & 6.18 & 0.76 \\
\hline$\mu$ & 4.48 & 4.9 & 5.38 & 1.42 & & \\
\hline $9.00-9.30$ & $\mathrm{Ta}$ & tc & $\mathrm{Td}$ & $\mathrm{Tb}$ & $\mathrm{Xi}-\mu$ & $\mathrm{Xi}-\mu$ \\
\hline 1 & 3.24 & 1.84 & 4.02 & 0.58 & 0.13 & 1.358 \\
\hline 2 & 1.20 & 2.86 & 2.98 & 2.42 & 2.17 & 0.482 \\
\hline 3 & 3.30 & 3.48 & 1.14 & 1.80 & 0.07 & 0.138 \\
\hline 4 & 3.86 & 1.34 & 2.94 & 0.76 & 0.49 & 1.178 \\
\hline 5 & 2.56 & 1.46 & 0.84 & 2.98 & 0.81 & 1.042 \\
\hline 6 & 3.82 & 1.26 & 0.90 & 1.46 & 0.45 & 0.478 \\
\hline 7 & 3.80 & 4.08 & 1.00 & 1.54 & 0.43 & 0.398 \\
\hline 8 & 4.06 & 2.24 & 3.26 & 2.62 & 0.69 & 0.682 \\
\hline 9 & 3.80 & 3.80 & 3.86 & 0.54 & 0.43 & 1.398 \\
\hline 10 & 4.06 & 1.12 & 2.22 & 4.68 & 0.69 & 2.742 \\
\hline$\sum$ & 33.70 & 23.48 & 23.16 & 19.38 & 3.67 & 4.198 \\
\hline$\mu$ & 3.37 & 2.348 & 2.316 & 1.938 & & \\
\hline
\end{tabular}

Table 2.3 (road 2/2 UD) the headway between the car (Ta), car and motorcycle (Tc), motorcycle and car (Td) and motorcycle and motorcycle (Tb) in the morning (peak hour) is higher than during the day, the analysis shows the traffic volume in the morning is not different significantly with during the day.

Table 2.4 (road 6/2 D) the headway between the car (Ta), car and motorcycle (Tc), motorcycle and car (Td) and motorcycle and motorcycle $(\mathrm{Tb})$ in the morning (peak hour) is higher than during the day, the analysis shows the traffic volume in the morning is not different significantly with during the day.

From Table $2.1-2.8$ we can see that headway time variation based on type of road and the location of road, those headway variation determine the value passenger car unit for motor cycle. 
Table 2.4. Pasteur Road 6/2 D

\begin{tabular}{|c|c|c|c|c|c|c|}
\hline Periode & $\mathrm{Ta}$ & $\mathrm{Tc}$ & $\mathrm{Td}$ & $\mathrm{Tb}$ & $\mathrm{ta}$ & $\mathrm{tb}$ \\
\hline $7.30-8.00$ & $\mathrm{Mb}-\mathrm{Mb}$ & $\mathrm{Mb}-\mathrm{Mt}$ & $\mathrm{Mt}-\mathrm{Mb}$ & $\mathrm{Mt}-\mathrm{Mt}$ & $\mathrm{Xi}-\mu$ & $\mathrm{Xi}-\mu$ \\
\hline 1 & 6.98 & 1.20 & 2.12 & 1.14 & 2.823 & 0.285 \\
\hline 2 & 3.62 & 1.10 & 1.74 & 0.75 & 0.537 & 0.675 \\
\hline 3 & 4.46 & 1.53 & 2.43 & 1.20 & 0.303 & 0.225 \\
\hline 4 & 3.87 & 3.26 & 1.62 & 1.41 & 0.282 & 0.015 \\
\hline 5 & 4.10 & 2.43 & 1.94 & 1.35 & 0.057 & 0.075 \\
\hline 6 & 2.48 & 1.31 & 4.22 & 1.05 & 1.677 & 0.375 \\
\hline 7 & 4.16 & 1.22 & 3.21 & 3.53 & 0.003 & 2.1 \\
\hline 8 & 5.00 & 1.46 & 0.86 & 1.58 & 0.843 & 0.15 \\
\hline 9 & 2.06 & 2.27 & 2.13 & 0.83 & 2.097 & 0.6 \\
\hline 10 & 4.83 & 1.61 & 2.70 & 1.43 & 0.678 & $2.22 \mathrm{E}-16$ \\
\hline$\Sigma$ & 41.52 & 17.36 & 22.95 & 14.25 & 4.002 & 1.275 \\
\hline$\mu$ & 4.152 & 1.7355 & 2.295 & 1.425 & & \\
\hline & & & & & ta & tb \\
\hline $9.00-9.30$ & $\mathrm{Ta}$ & $\mathrm{Tc}$ & $\mathrm{Td}$ & $\mathrm{Tb}$ & $\mathrm{Xi}-\mu$ & $\mathrm{Xi}-\mu$ \\
\hline 1 & 4.2 & 1.84 & 4.02 & 1.58 & 0.92 & 0.658 \\
\hline 2 & 3.8 & 2.86 & 2.98 & 2.42 & 0.52 & 0.182 \\
\hline 3 & 4.4 & 3.48 & 1.14 & 1.8 & 1.12 & 0.438 \\
\hline 4 & 2.2 & 1.34 & 2.94 & 1.76 & 1.08 & 0.478 \\
\hline 5 & 3.8 & 1.46 & 0.84 & 2.98 & 0.52 & 0.742 \\
\hline 6 & 3.8 & 1.26 & 0.9 & 1.46 & 0.52 & 0.778 \\
\hline 7 & 2.4 & 4.08 & 1 & 1.54 & 0.88 & 0.698 \\
\hline 8 & 3.2 & 2.24 & 3.26 & 2.62 & 0.08 & 0.382 \\
\hline 9 & 1.4 & 3.8 & 3.86 & 1.54 & 1.88 & 0.698 \\
\hline 10 & 3.6 & 1.12 & 2.22 & 4.68 & 0.32 & 2.442 \\
\hline$\Sigma$ & 32.80 & 23.48 & 23.16 & 22.38 & 4.16 & 2.498 \\
\hline$\mu$ & 3.28 & 2.348 & 2.316 & 2.238 & & \\
\hline & & & & & & \\
\hline
\end{tabular}

Table 2.5. Soegijopranoto Road 6/2 UD

\begin{tabular}{|c|c|c|c|c|c|c|}
\hline Periode & $\mathrm{Ta}$ & $\mathrm{Tc}$ & $\mathrm{Td}$ & $\mathrm{Tb}$ & $\mathrm{Ta}$ & $\mathrm{Tb}$ \\
\hline $8.00-8.30$ & $\mathrm{Mb}-\mathrm{Mb}$ & $\mathrm{Mb}-\mathrm{Mt}$ & $\mathrm{Mt}-\mathrm{Mb}$ & $\mathrm{Mt}-\mathrm{Mt}$ & $\mathrm{Xi}-\mu$ & $\mathrm{Xi}-\mu$ \\
\hline 1 & 15 & 3 & 5 & 3 & 0.3 & 0.5 \\
\hline 2 & 13.5 & 6 & 8 & 2 & 1.2 & 0.5 \\
\hline 3 & 14 & 15 & 4 & 3 & 0.7 & 0.5 \\
\hline 4 & 15 & 8 & 9 & 2.5 & 0.3 & 0 \\
\hline 5 & 16 & 16 & 12 & 2 & 1.3 & 0.5 \\
\hline$\sum$ & 73.5 & 48 & 38 & 12.5 & 3.8 & 2 \\
\hline$\mu$ & 14.7 & 9.6 & 7.6 & 2.5 & & \\
\hline & & & & & $\mathrm{Ta}$ & $\mathrm{Tb}$ \\
\hline $9.00-9.30$ & $\mathrm{Ta}$ & $\mathrm{Tc}$ & $\mathrm{Td}$ & $\mathrm{Tb}$ & $\mathrm{Xi}-\mu$ & $\mathrm{Xi}-\mu$ \\
\hline 1 & 25 & 15 & 11 & 4 & 3.8 & 0.6 \\
\hline 2 & 22 & 14 & 15 & 4 & 0.8 & 0.6 \\
\hline 3 & 20 & 18 & 13 & 3 & 1.2 & 0.4 \\
\hline 4 & 22 & 17 & 14 & 3 & 0.8 & 0.4 \\
\hline 5 & 17 & 19 & 13 & 3 & 4.2 & 0.4 \\
\hline$\sum$ & 106 & 83 & 66 & 17 & 10.8 & 2.4 \\
\hline$\mu$ & 21.2 & 16.6 & 13.2 & 3.4 & & \\
\hline
\end{tabular}


Table 2.6. Thamrin Road 4/2 D

\begin{tabular}{|c|c|c|c|c|c|c|}
\hline Periode & Ta & Tc & $\mathrm{Td}$ & $\mathrm{Tb}$ & ta & $\mathrm{Tb}$ \\
\hline $7.30-8.00$ & $\mathrm{Mb}-\mathrm{Mb}$ & $\mathrm{Mb}-\mathrm{Mt}$ & Mt-Mb & Mt-Mt & $\mathrm{Xi}-\mu$ & $\mathrm{Xi}-\mu$ \\
\hline 1 & 1.43 & 1.22 & 1.71 & 1.41 & 3.56 & 0.284 \\
\hline 2 & 1.77 & 0.34 & 3.1 & 2.21 & 3.22 & 1.084 \\
\hline 3 & 0.52 & 0.37 & 1.11 & 1.24 & 4.47 & 0.114 \\
\hline 4 & 0.59 & 0.35 & 0.31 & 2.12 & 4.4 & 0.994 \\
\hline 5 & 4.3 & 2.52 & 1.12 & 0.34 & 0.69 & 0.786 \\
\hline 6 & 1.74 & 1.16 & 1.25 & 1.15 & 3.25 & 0.024 \\
\hline 7 & 4.06 & 2.9 & 1.61 & 1.32 & 0.93 & 0.194 \\
\hline 8 & 2.11 & 1.74 & 0.31 & 0.21 & 2.88 & 0.916 \\
\hline 9 & 2.78 & 1.72 & 0.46 & 0.23 & 2.21 & 0.896 \\
\hline 10 & 30.6 & 0.47 & 3.12 & 1.03 & 25.61 & 0.096 \\
\hline$\sum$ & 49.90 & 12.79 & 14.10 & 11.26 & 16.34 & 3.262 \\
\hline$\mu$ & 4.99 & 1.278833 & 1.41 & 1.126 & & \\
\hline $9.00-9.30$ & $\mathrm{Ta}$ & $\mathrm{Tc}$ & $\mathrm{Td}$ & $\mathrm{Tb}$ & $\mathrm{Xi}-\mu$ & $\mathrm{Xi}-\mu$ \\
\hline 1 & 1.46 & 1.71 & 0.52 & 0.5 & 0.733 & 0.254 \\
\hline 2 & 1.7 & 1.8 & 1.45 & 0.39 & 0.493 & 0.364 \\
\hline 3 & 1.88 & 1.42 & 0.89 & 0.67 & 0.313 & 0.084 \\
\hline 4 & 1.43 & 0.32 & 0.72 & 0.46 & 0.763 & 0.294 \\
\hline 5 & 1.32 & 1.88 & 0.52 & 0.89 & 0.873 & 0.136 \\
\hline 6 & 1.77 & 0.31 & 0.5 & 0.63 & 0.423 & 0.124 \\
\hline 7 & 1.8 & 0.98 & 1.51 & 0.35 & 0.393 & 0.404 \\
\hline 8 & 2.11 & 1.24 & 1.46 & 1.44 & 0.083 & 0.686 \\
\hline 9 & 4.27 & 0.45 & 1.34 & 1.62 & 2.077 & 0.866 \\
\hline 10 & 4.19 & 0.8 & 1.15 & 0.59 & 1.997 & 0.164 \\
\hline$\sum$ & 21.93 & 10.91 & 10.06 & 7.54 & 3.175 & 1.132 \\
\hline$\mu$ & 2.193 & 1.091 & 1.006 & 0.754 & & \\
\hline
\end{tabular}


Table 2.7. Pandanaran Road 8/2 D

\begin{tabular}{|c|c|c|c|c|c|c|}
\hline Periode & $\mathrm{Ta}$ & tc & $\mathrm{Td}$ & $\mathrm{Tb}$ & ta & $\mathrm{Tb}$ \\
\hline $7.30-8.00$ & $\mathrm{Mb}-\mathrm{Mb}$ & $\mathrm{Mb}-\mathrm{Mt}$ & $\begin{array}{l}\text { Mt- } \\
\mathrm{Mb}\end{array}$ & Mt-Mt & $\mathrm{Xi}-\mu$ & $X i-\mu$ \\
\hline 1 & 3.96 & 2.76 & 4.7 & 3.48 & 0.012 & 0.48 \\
\hline 2 & 4.1 & 3.38 & 3.28 & 2.76 & 0.152 & 0.24 \\
\hline 3 & 3.88 & 3.42 & 4.42 & 3.08 & 0.068 & 0.08 \\
\hline 4 & 5.5 & 3.38 & 3.86 & 1.86 & 1.552 & 1.14 \\
\hline 5 & 5.76 & 3.06 & 4.66 & 2.7 & 1.812 & 0.3 \\
\hline 6 & 4.54 & 2.34 & 4.36 & 3.1 & 0.592 & 0.1 \\
\hline 7 & 4.2 & 2.6 & 3.62 & 2.52 & 0.252 & 0.48 \\
\hline 8 & 2.34 & 3.54 & 4.42 & 3.64 & 1.608 & 0.64 \\
\hline 9 & 1.82 & 3.06 & 4.04 & 3.2 & 2.128 & 0.2 \\
\hline 10 & 3.38 & 3.68 & 4.52 & 3.66 & 0.568 & 0.66 \\
\hline$\sum$ & 39.48 & 31.22 & 41.88 & 30.00 & 3.596 & 2.24 \\
\hline \multirow[t]{2}{*}{$\mu$} & 3.948 & 3.122 & 4.188 & 3 & & \\
\hline & & & & & ta & $\mathrm{Tb}$ \\
\hline $9.00-9.30$ & ta & tc & $\mathrm{td}$ & $\mathrm{Tb}$ & $X i-\mu$ & $\mathrm{Xi}-\mu$ \\
\hline 1 & 3.16 & 0.84 & 4.32 & 2.14 & 0.882 & 0.498 \\
\hline 2 & 3.38 & 5.66 & 1.02 & 2.68 & 0.662 & 0.042 \\
\hline 3 & 7.2 & 1.9 & 2.28 & 2.8 & 3.158 & 0.162 \\
\hline 4 & 2.24 & 1.96 & 1.54 & 2.8 & 1.802 & 0.162 \\
\hline 5 & 4.64 & 2.24 & 1.04 & 2.32 & 0.598 & 0.318 \\
\hline 6 & 3.14 & 2 & 2.28 & 2.44 & 0.902 & 0.198 \\
\hline 7 & 4.38 & 2.34 & 2.14 & 3.4 & 0.338 & 0.762 \\
\hline 8 & 4.88 & 1.2 & 1.32 & 2.6 & 0.838 & 0.038 \\
\hline 9 & 4.14 & 1.34 & 2.82 & 3.4 & 0.098 & 0.762 \\
\hline 10 & 3.26 & 1.56 & 2.46 & 1.8 & 0.782 & 0.838 \\
\hline$\Sigma$ & 40.42 & 21.04 & 21.22 & 26.38 & 7.102 & 1.182 \\
\hline$\mu$ & 4.042 & 2.104 & 2.122 & 2.638 & & \\
\hline
\end{tabular}


Table 2.8 Jalan Imam Bonjol 2/2 UD

\begin{tabular}{|c|c|c|c|c|c|c|}
\hline Periode & ta & $\mathrm{Tc}$ & $\mathrm{Td}$ & $\mathrm{Tb}$ & ta & $\mathrm{tb}$ \\
\hline $7.30-8.00$ & $\mathrm{Mb}-\mathrm{Mb}$ & $\mathrm{Mb}-\mathrm{Mt}$ & $\mathrm{Mt}-\mathrm{Mb}$ & Mt-Mt & $\mathrm{Xi}-\mu$ & $\mathrm{Xi}-\mu$ \\
\hline 1 & 4.65 & 0.8 & 1.41 & 0.76 & 1.882 & 0.19 \\
\hline 2 & 2.41 & 0.73 & 0.49 & 0.5 & 0.358 & 0.45 \\
\hline 3 & 2.97 & 1.02 & 1.62 & 0.8 & 0.202 & 0.15 \\
\hline 4 & 2.58 & 2.17 & 1.08 & 0.94 & 0.188 & 0.01 \\
\hline 5 & 2.73 & 1.62 & 1.29 & 0.9 & 0.038 & 0.05 \\
\hline 6 & 1.65 & 0.87 & 2.81 & 0.7 & 1.118 & 0.25 \\
\hline 7 & 2.77 & 0.81 & 2.14 & 2.35 & 0.002 & 1.4 \\
\hline 8 & 3.33 & 0.97 & 0.24 & 1.05 & 0.562 & 0.1 \\
\hline 9 & 1.37 & 1.51 & 1.42 & 0.55 & 1.398 & 0.4 \\
\hline 10 & 3.22 & 1.07 & 3.8 & 0.95 & 0.452 & 1.16 \\
\hline$\sum$ & 27.68 & 11.57 & 16.30 & 9.50 & 2.668 & 0.85 \\
\hline$\mu$ & 2.768 & 1.157 & 1.63 & 0.95 & & \\
\hline $9.00-9.30$ & ta & $\mathrm{Tc}$ & $\mathrm{Td}$ & $\mathrm{Tb}$ & $\mathrm{Xi}-\mu$ & $\mathrm{Xi}-\mu$ \\
\hline 1 & 1.62 & 0.92 & 2.01 & 0.29 & 0.065 & 0.679 \\
\hline 2 & 0.6 & 1.43 & 1.49 & 1.21 & 1.085 & 0.241 \\
\hline 3 & 1.65 & 1.74 & 0.57 & 0.9 & 0.035 & 0.069 \\
\hline 4 & 1.93 & 0.67 & 1.47 & 0.38 & 0.245 & 0.589 \\
\hline 5 & 1.28 & 0.73 & 0.42 & 1.49 & 0.405 & 0.521 \\
\hline 6 & 1.91 & 0.63 & 0.45 & 0.73 & 0.225 & 0.239 \\
\hline 7 & 1.9 & 2.04 & 0.5 & 0.77 & 0.215 & 0.199 \\
\hline 8 & 2.03 & 1.12 & 1.63 & 1.31 & 0.345 & 0.341 \\
\hline 9 & 1.9 & 1.9 & 1.93 & 0.27 & 0.215 & 0.699 \\
\hline 10 & 2.03 & 0.56 & 1.11 & 2.34 & 0.345 & 1.371 \\
\hline$\sum$ & 16.85 & 11.74 & 11.58 & 9.69 & 1.835 & 2.099 \\
\hline$\mu$ & 1.685 & 1.174 & 1.158 & 0.969 & & \\
\hline
\end{tabular}

\section{Data analysis}

\subsection{PCU calculation}

The results of calculation of the value PCU across the road at the morning peak hours and off-peak hours of daylight are presented in Table 3.1 and Table 3.2 below:

Table 3.1 Summary of value PCU at Bandung

\begin{tabular}{|l|l|c|c|}
\hline \multirow{3}{*}{ Metode } & \multirow{2}{*}{ Observation Location } & \multicolumn{2}{|c|}{ PCU } \\
\cline { 3 - 4 } & & \multicolumn{2}{|c|}{ Motor Cycle } \\
\cline { 3 - 4 } & & Peak & Off Peak \\
\hline \multirow{3}{*}{ Headway } & Pasteur & 0,28 & 0,33 \\
\cline { 2 - 4 } & Dipati Ukur & 0,22 & 0,26 \\
\cline { 2 - 4 } & Juanda & 0,24 & 0,33 \\
\cline { 2 - 4 } & Tubagus Ismail & 0.18 & 0,19 \\
\hline
\end{tabular}


From the analysis of PCU obtained from four locations in Bandung area PCU Motorcycles values obtained varied from 0.18 to 0.28 during peak hours and the value of PCU Motorcycles at off peak hours vary from 0.19 to 0.33 .

Table 3.2 Summary of value PCU at Semarang

\begin{tabular}{|l|l|c|c|}
\hline \multirow{3}{*}{ Metode } & \multirow{2}{*}{ Observation Location } & \multicolumn{2}{|c|}{ PCU } \\
\cline { 3 - 4 } & & \multicolumn{2}{|c|}{ Motor Cycle } \\
\cline { 3 - 4 } & Soegijopranoto & Peak & Off Peak \\
\hline \multirow{5}{*}{ Headway } & Thamrin & 0,29 & 0,34 \\
\cline { 2 - 4 } & Padanaran & 0,24 & 0,33 \\
\cline { 2 - 4 } & Imam Bonjol & 0,29 & 0,35 \\
\hline
\end{tabular}

From the analysis of PCU obtained from four locations in Semarang area PCU Motorcycles values obtained varied from 0.19 to 0.29 during peak hours and the value of PCU Motorcycles at off peak hours vary from 0.22 to 0.35 .

\subsection{RMSE analysis of PCU value}

Comparisson between data at road of Bandung and data at road of Semarang on off peak hour use Root Mean Suare Error (RMSE) Analysis describe at Table 6.3 below :

Table 3.3 RMSE on peak hour

\begin{tabular}{|c|c|c|c|c|c|c|}
\hline \multirow{2}{*}{ ROAD TYPE } & \multicolumn{3}{|c|}{ PCU VALUE } & \multicolumn{3}{c|}{ Difference (RMSE) } \\
\cline { 2 - 7 } & IHCM & BANDUNG & SEMARANG & $2-3$ & $2-4$ & $4-3$ \\
\hline 1 & 2 & 3 & 4 & 5 & 6 & 7 \\
\hline $6 / 2 \mathrm{D}$ & 0.4 & 0.33 & 0.35 & $53 \%$ & $18 \%$ & $42 \%$ \\
\hline $6 / 2$ UD & - & - & 0.34 & - & - & - \\
\hline $4 / 2 \mathrm{D}$ & 0.4 & 0.33 & 0.33 & $58 \%$ & $18 \%$ & $48 \%$ \\
\hline 4/2 UD & 0.4 & 0.26 & - & $60 \%$ & - & - \\
\hline 2/2 UD & 0.4 & 0.19 & 0.22 & $45 \%$ & $53 \%$ & $16 \%$ \\
\hline Average & - & - & - & 0.54 & 0.30 & 0.35 \\
\hline
\end{tabular}

Comparisson between data at road of Bandung and data at road of Semarang on peak hour describe at Table 6.4.

Table 3.4 RMSE off peak hour

\begin{tabular}{|c|c|c|c|c|c|c|}
\hline \multirow{2}{*}{ ROAD TYPE } & \multicolumn{3}{|c|}{ PCU VALUE } & \multicolumn{3}{c|}{ Difference (RMSE) } \\
\cline { 2 - 7 } & IHCM & BANDUNG & SEMARANG & $2-3$ & $2-4$ & $4-3$ \\
\hline 1 & 2 & 3 & 4 & 5 & 6 & 7 \\
\hline $6 / 2 \mathrm{D}$ & 0.25 & 0.28 & 0.29 & $12 \%$ & $12 \%$ & $21 \%$ \\
\hline $6 / 2$ UD & - & - & 0.29 & & & \\
\hline $4 / 2 \mathrm{D}$ & 0.25 & 0.24 & 0.24 & $16 \%$ & $4 \%$ & $13 \%$ \\
\hline $4 / 2$ UD & 0.25 & 0.22 & - & $12 \%$ & $12 \%$ & $0 \%$ \\
\hline $2 / 2$ UD & 0.25 & 0.18 & 0.19 & $24 \%$ & $28 \%$ & $6 \%$ \\
\hline Average & - & - & - & 0.16 & 0.14 & 0.13 \\
\hline
\end{tabular}

PCU value based on survey in Bandung and Semarang show that at the Off Peak Hour the PCU value of both cities more different with PCU based on IHCM compare at the Peak Hour. It means PCU value version IHCM is more not appropriate when off peak hour condition. 
Based total population and density population shows the PCU of motor cycle looks influenced by total population or density population, which PCU of motor cycle in Bandung looks smaller than in Semarang (Bandung has more population than Semarang).

\section{Conclusions and recommendations}

\subsection{Conclusion}

1. Value PCU Motor cycles based on research results on average smaller than the value PCU based IHCM good at peak hour and off-peak hours.

2. At off peak hour the difference between PCU based on survey and PCU based on IHCM greater than at peak hour.

3. PCU value version IHCM is more not appropriate when off peak hour condition.

4. The density of traffic significantly influence the PCU value of motor cycle.

5. The traffic characteristic for same road type is different between Bandung and Semarang.

6. Total Population or Density Population in City influence PCU value of motorcycle, so more total population or density population shows bigger PCU of motorcycle.

\subsection{Recomendation}

1. Need to look at the relationship PCU value on the level of service roads on each road

2. The studies should be done on a smaller city or town has a population of fewer than Bandung and Semarang.

\section{References}

1. Nur, N.K., Samang, L., Isran Ramli, M., Hamid, S., Study of modes transformation preferences of private transport based on travel characteristics and user behavior. International Journal of Applied Engineering Research. Vol. 11(22), pp. 10766-10771. (2016)

2. Directorate General Bina Marga (2010), Road Classification in Indonesia, Directorate General of Highways, Ministry of Public Works Indonesia.

3. IHCM (1997), Indonesia Highway Capacity Manual, Directorate General of Highways, Ministry of Public Works Indonesia.

4. Izumi Okura, (2006), Determination value of Passenger Car Equivalent (PCE)

5. Rosma I (2003), Determining PCU Value for Motorcycle at Intersection in Surakarta, UNS University.

6. Central Statistic Bureau (2015)

7. Scraggs, D.A (1964), determination of the passenger car eqivalent of a good vehicle in single lane flow at traffic signals, U.K., Road Research laboratory, Harmondsworth and Langley. 